\title{
Study on the Mechanism of Periplaneta americana Extract to Accelerate Wound Healing after Diabetic Anal Fistula Operation Based on Network Pharmacology
}

\author{
Fengfei Wang $\mathbb{D}^{1},{ }^{1}$ Shuai $\mathrm{Li}^{2},{ }^{2}$ Le $\mathrm{Ma},{ }^{3}$ Yuefei Geng, ${ }^{4}$ Yongmei Shen, ${ }^{4}$ and Juanni Zeng $\mathbb{D}^{1}$ \\ ${ }^{1}$ The Second Hospital of Hunan University of Traditional Chinese Medicine, Hunan, Changsha 410005, China \\ ${ }^{2}$ Hunan University of Traditional Chinese Medicine, Hunan, Changsha 410208, China \\ ${ }^{3}$ The First Hospital of Hunan University of Traditional Chinese Medicine, Hunan, Changsha 410007, China \\ ${ }^{4}$ Sichuan Key Laboratory of Medical American Cockroach, Sichuan, Chengdu 610000, China \\ Correspondence should be addressed to Juanni Zeng; 575826199@qq.com
}

Received 22 October 2020; Revised 15 February 2021; Accepted 25 February 2021; Published 10 March 2021

Academic Editor: Mohammad Hashem Hashempur

Copyright (C) 2021 Fengfei Wang et al. This is an open access article distributed under the Creative Commons Attribution License, which permits unrestricted use, distribution, and reproduction in any medium, provided the original work is properly cited.

\begin{abstract}
Objective. Using network pharmacology research methods to explore the healing mechanism of American cockroach extract to accelerate wound healing after diabetic anal fistula surgery. Method. The main chemical constituents of extracts from Periplaneta americana were collected by literature retrieval. Chemical composition and targets related to diabetic anal fistula wound could be predicted based on PubChem, Swiss Target Prediction, OMIM, and GeneCards databases, and the putative targets of Periplaneta americana extraction (PAE) for diabetic anal fistula wound were obtained by Venn diagram. These common targets were predicted using the String database for protein-protein interaction (PPI) network and then screening key genes through Cytohubba. Meanwhile, the above targets were analyzed using the DAVID database for gene ontology (GO) enrichment analyses and the Kyoto Encyclopedia of Genes and Genomes (KEGG) path enrichment analyses. Results. A total of 12 chemical components of PAE were obtained by literature retrieval, and 61 therapeutic targets that may accelerate the healing of diabetic anal fistula wounds were predicted by the database. According to PPI network analysis, PAE accelerates wound healing after diabetic anal fistula surgery which may be related to proteins such as AKT1, VEGFA, EGFR, CASP3, STAT3, MAPK1, TNF, JUN, ESR1, and MMP9. GO analysis results show that targets of PAE to promote wound healing were mainly involved in biological processes such as cell proliferation, macrophage differentiation, angiogenesis, and response to hypoxia. KEGG analysis showed that the target genes were mainly concentrated in the PI3K-Akt signaling pathway, HIF-1 signaling pathway, and estrogen signaling pathway. Conclusion. Periplaneta americana extract regulates multiple targets and multiple pathways to promote wound healing after diabetic anal fistula surgery. PI3K-Akt signaling pathway, HIF-1 signaling pathway, and sex hormone signaling pathway may be key pathways in the process of Periplaneta americana extract promoting wound healing.
\end{abstract}

\section{Introduction}

Anal fistula is a tube located between the perianal skin and the rectum. It is caused by chronic infection and epithelialization of the drainage tube [1]. It is one of the common diseases in the anorectal department, and surgery is the first choice. Epidemiological data show that the total number of diabetes patients in the world was 463 million in 2019, and it is estimated that it will rise to 578 million in 2030 and 700 million in 2045 [2]. Prolonged generation of pathological blood sugar elevation in the body can lead to the sequel of advanced glycation end products in the body, which provide a good nutritional environment for the growth of bacteria, and easily cause wound tissue infection and necrosis, and hinder wound healing. Diabetes is a risk factor leading to slow wound healing after anal fistula surgery [3-5].

In wound healing, some herbal and animal Chinese medicines have very good clinical effects [6-8], and Quyushengxin formula can promote mucosal healing in UC patients [9]. In traditional Persian medicine [10], the plants' hard tissues such as roots or barks were boiled in water. Subsequently, the resulting extract was boiled in 
combination with sesame or olive oil until its water part was lost to exert its efficacy. Periplaneta americana (PA), the American cockroach, has the largest body size in the family Blattidae. It is an animal traditional Chinese medicine. Physiological and pharmacological studies have demonstrated that PA constituents have favorable tissue-repairing, antibacterial, antitumor, and immunity-enhancing activities [11]. Additionally, this insect has been widely used for the treatment of various wounds, ulcers, fistulas, bedsores, and burns [12]. Consequently, the formulation of many TCM preparations, among them, Kangfuxin (Periplaneta americana extract, $\mathrm{PAE}$ ), is a liquid preparation that includes ingredients from PA that has been used to treat different skin or mucosa injuries in China for more than 40 years. Our team applied PAE to wounds after anorectal diseases in the early years and found that the effect of promoting wound healing was very good [13]. Besides, it has been reported that $\mathrm{PAE}$ can promote postoperative wound healing in diabetic patients [14], but there are few studies on postoperative wounds in diabetes patients with anal fistula. We hypothesize that PAE can promote postoperative wound healing in diabetes patients with anal fistula, and its mechanism for promoting healing remains to be further studied.

Network pharmacology is an emerging research method first proposed by Andrew L Hopkins in Nature Biotechnology in 2007 [15]. It is based on drug databases, disease databases, gene pleiotropy, computational biology, and network biology analysis, etc., to construct a "drug-targetdisease-pathway" interaction network and to comprehensively analyze the relationship of drug components, genes, and diseases, thereby revealing the intervention mechanism of drugs on the disease. Its holistic and dynamic characteristics, the principle of multitarget, and multichannel administration are highly consistent with the holistic view of traditional Chinese medicine and the treatment principle of syndrome differentiation. The purpose of this study is to use the research methods of network pharmacology to initially find the target of PAE in repairing postoperative wounds of diabetes patients with anal fistula and to provide a theoretical basis for further application.

\section{Materials and Methods}

2.1. Effective Chemical Ingredient Screening. There are rarely studies on the chemical components of Periplaneta americana, and no relevant chemical ingredients are included in the TCMSP database (http://ibts.hkbu.edu.hk/LSP/Tcmsp. php). We use "American Cockroach (Periplaneta americana)," "KANG FU XIN YE," as the keyword to retrieve in the PubMed and CNKI databases. 12 compounds related to wound healing were screened out through literature research [11, 12, 16]: cytosine, cytidine, thymine, uracil, guanosine, adenine, hypoxanthine, inosine, uridine, cyclo-(L-Val-LPro), arbutin, and (E)-3-hexenyl- $\beta$-D-glucopyranoside.

2.2. Target Prediction of Drug. The compound was imported into PubChem (https://pubchem.ncbi.nlm.nih.gov/) to obtain SMILES, and the Swiss Target Prediction database (http://www.swisstargetprediction.ch/) was used to predict potential targets and limit the species: Homo sapiens. The target point of the drug component was obtained.

2.3. Drug-Disease-Related Target Screening. Disease targets are derived from the Human Mendelian Genetic Database (OMIM) (http://www.omim.org/) and the human genome annotation database (GeneCards) (https://www.genecards. org/) with the keywords "diabetic anal fistula wound." Then, the genes of the two databases were integrated to remove the duplicates to obtain the relevant targets of diabetic anal fistula wounds. Finally, the Venn diagram was used to find out the intersection genes of disease target genes and drug target genes, that is, the potential target of American cockroach extract in treating diabetic anal fistula wounds.

\subsection{Network Construction and Analyses}

2.4.1. Construction of the Compound-Target Network. The drug components of PAE and their potential targets for promoting wound healing after diabetic anal fistula surgery were introduced into Cytoscape 3.1.1 to construct a network diagram of active ingredients and disease targets. The network was analyzed through the network analysis function. The degree, closeness, and betweenness are important parameters for evaluating active ingredients and targets.

\subsubsection{Construction of Protein-Protein Interaction Network.} The intersection gene was uploaded to the String database (https://string-db.org/), the species were limited to "Homo sapiens," the minimum interaction threshold was set to "medium confidence" 0.4 , and nodes without network connections were hidden. The protein-protein interaction (PPI) network diagram was obtained. The Cytoscape 3.1.1 software was used to realize the visualization of the network, and then, the Cytohubba plug-in was used to screen the top 10 key genes according to degree.

2.4.3. GO and KEGG Analyses. The potential targets of PAE in promoting wound healing after diabetic anal fistula surgery were analyzed by Gene Ontology enrichment and Kyoto Encyclopedia of Genes and Genomes pathway enrichment in the Database for Annotation, Visualization, and Integrated Discovery (DAVID) v6.8, $p$ value $<0.05$, and the species selected were "Homo sapiens," elucidating the potential healing mechanism of American cockroach extract in the treatment of diabetic anal fistula wounds.

\section{Results}

3.1. Targets of Active Ingredients in Periplaneta americana. There are a total of 797 predicted targets for 12 compounds, including 6 targets in cytosine, 100 in cytidine, 19 in thymine, 25 in uracil, 100 in guanosine, 32 in adenine, 32 targets in hypoxanthine, 100 in inosine, 100 in uridine, 100 in cyclo(L-Val-L-Pro), 86 in arbutin targets, and 97 targets in (E)-3hexenyl- $\beta$-D-glucopyranoside. The 12 compound target 
genes were merged, and then the duplicate genes were deleted to obtain 370 drug targets.

3.2. Periplaneta americana Treatment Targets for Diabetic Anal Fistula Wounds. 693 targets related to diabetic anal fistula wounds were retrieved through OMIM and GeneCards disease databases. 61 targets for the treatment of diabetic anal fistula wounds in Periplaneta americana were obtained by the Venn diagram online tool (Figure 1).

3.3. Compound-Target Network. Based on 61 common target genes as targets for the treatment of diabetic anal fistula wounds, a network diagram of drug component targets was established (Figure 2). In this network diagram, nodes represent target genes and drug components, and edges represent the interaction between drug components and target genes. There are 72 nodes and 128 edges in total. The 12 active ingredients of Periplaneta americana act on the same or different targets. The network diagram fully reflects the multicomponent and multitarget mechanism of PAE to promote wound healing after diabetic anal fistula surgery. Through network topology analysis, the average degree of the network graph is 3.5 , the average closeness is 0.3 , and the average betweenness is 0.03 . For 9 components, cyclo-(LVal-L-Pro), (E)-3-hexenyl- $\beta$-D-glucopyranoside, arbutin, uridine, cytidine, inosine, guanosine, adenine, and uracil, the values of degree, closeness, and betweenness are all greater than the average, suggesting that these active ingredients of Periplaneta Americana play an important role in the treatment of diabetic anal fistula wounds. See the main parameter values in Table 1.

3.4. PPI Network Analysis. The target protein interaction network was analyzed by the online website STRING. Therefore, we obtained a protein interaction network of 552 interaction relationships with 60 nodes (Figure 3(a)) and calculated that the node "connectivity" of the network was 18.1 on average. By using the Cytohubba analysis tool, the top 10 hub genes were obtained according to degree, and the results show that the hub genes are AKT1, VEGFA, EGFR, CASP3, STAT3, MAPK1, TNF, JUN, ESR1, and MMP9, as shown in Figure $3(\mathrm{~b})$. There is a strong interaction relationship, which may be a potential key gene for American cockroach extract to promote diabetic anal fistula wound repair.

3.5. Gene Ontology Enrichment Analysis. We imported 61 potential target genes into the DAVID 6.8 database for GO analysis. GO analysis includes three aspects: molecular function (MF), cellular component (CC), and biological process (BP) to predict the main biological functions performed by target genes. Under the criterion of $p<0.05$, the top 20 terms in the ranking are displayed. The results showed that BP of the target genes (Figure 4(a)) mainly occurs in the positive regulation of cell proliferation, nitric oxide biosynthesis, protein phosphorylation, and ERK1 and ERK2 cascades and shows the components involved in proteolytic



Figure 1: Target Venn diagram of Periplaneta americana and diabetic anal fistula wounds.

metabolism, macrophage differentiation, angiogenesis, and response to hypoxia. CC (Figure 4(b)) shows the components involved in nuclear chromosomes, organelles, and plasma membranes; MF (Figure 4(c)) mainly focuses on protein and zinc ion binding, protein tyrosine kinase activity, nitric oxide synthase regulatory activity, and guanosine regulation of nucleotide exchange factor activity.

3.6. KEGG Pathway Enrichment Analysis. KEGG pathway analysis was performed on 61 key target genes using the DAVID 6.8 database. Under the standard of $p<0.05,66$ signaling pathways were screened, which were arranged in ascending order according to the $p$ value. The enrichment results of the first 25 pathways showed that target genes were mainly enriched in the VEGF signaling pathway, PI3K-Akt signaling pathway, estrogen signaling pathway, MAPK signaling pathway, FoxO signaling pathway, and HIF-1 signaling pathway (Figure 4(d)). It is suggested that the above pathways may be involved in the regulation of diabetic anal fistula wound healing by Periplaneta americana.

\section{Discussion}

Wound healing goes through a continuous dynamic physiological process of inflammation, proliferation, and remodeling, involving a variety of cells and cytokines. Retention at any stage and failure to proceed in an orderly manner may cause delayed wound healing. In this study, a PPI network was constructed on the healing-promoting target of Periplaneta americana extract (PAE), and it was found that AKT1, VEGFA, EGFR, CASP3, STAT3, MAPK1, TNF, JUN, ESR1, and MMP9 interact closely. Our team's previous experimental studies have confirmed that the extract of Periplaneta americana maintains a high level of caspase-3 RNA expression in the early stage of wound healing, speeds up the shedding of necrotic tissue on the wound, and then slowly decreases the expression of water skin, reducing tissue hyperplasia and avoiding scar formation [17]; upregulating the expression of VEGF during the granulation phase of the wound to promote microangiogenesis in the ischemic area [18]; and downregulating the expression of Smad6 and upregulating the expression of Smad9, accelerating cell ultrastructural proliferation and differentiation, maintaining a stable internal environment of the cell, and accelerating wound tissue fibrosis [19]. It can increase the expression of type I/III collagen before chronic 


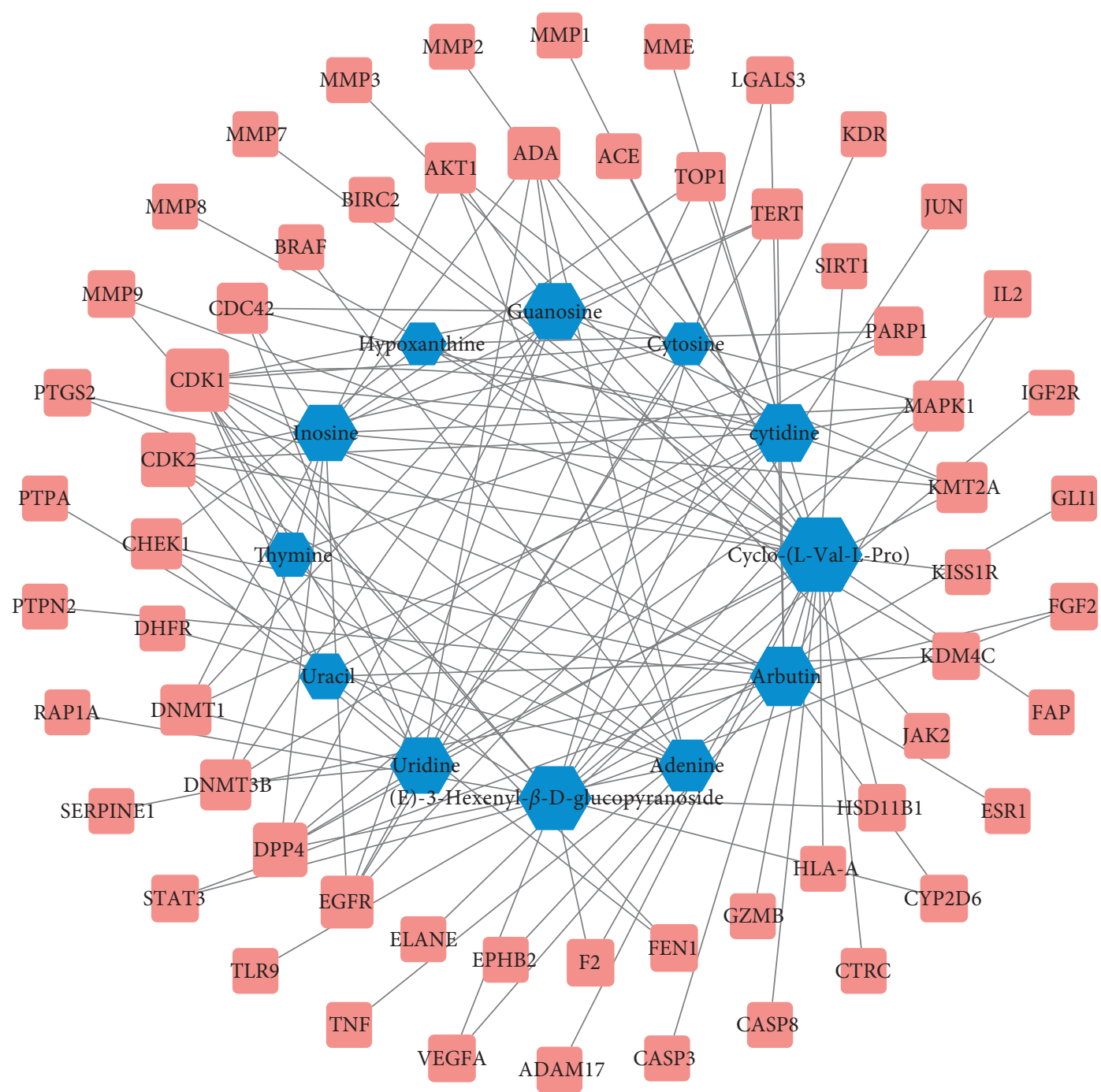

FIGURE 2: Blue represents the compound and red represents the target gene. The greater the degree value, the larger the node.

TABLE 1: Topological parameters of main ingredients.

\begin{tabular}{lccc}
\hline Node & Degree & Closeness & Betweenness \\
\hline Cyclo-(L-Val-L-Pro) & 24 & 0.45512821 & 0.4782475 \\
(E)-3-Hexenyl- $\beta$-D-glucopyranoside & 17 & 0.41764706 & 0.28230501 \\
Arbutin & 14 & 0.40804598 & 0.20036162 \\
Uridine & 12 & 0.3988764 & 0.06677689 \\
Cytidine & 12 & 0.39444444 & 0.05409036 \\
Inosine & 12 & 0.39444444 & 0.07432615 \\
Guanosine & 13 & 0.39444444 & 0.06911849 \\
Adenine & 9 & 0.38586957 & 0.11076596 \\
Uracil & 5 & 0.36979167 & 0.05474434 \\
\hline
\end{tabular}

wounds in rats to promote wound healing [20]. Lindner [21] found that AKT1 is involved in the regulation of defensor expression and an important innate immune response to bacterial clearance, which is particularly important in the early control of inflammation in chronic infectious wounds. Huang [22] designed in vivo and in vitro models. Compared with the control group, skin damage caused the upregulation of TNF- $\alpha$ expression in the peripheral blood of diabetic rats and a significant increase in M1 macrophage infiltration, which proved that the high-glucose environment that was caused by single nuclear cells is more prone to the polarization of M1 type macrophages. Yue [23] proposed that c-Jun overexpression promotes the proliferation and $\mathrm{mi}$ gration of human umbilical cord-derived mesenchymal stem cells (hUC-MSCs) in vitro and accelerates diabetic wound healing, re-epithelialization, and angiogenesis through hUC- 


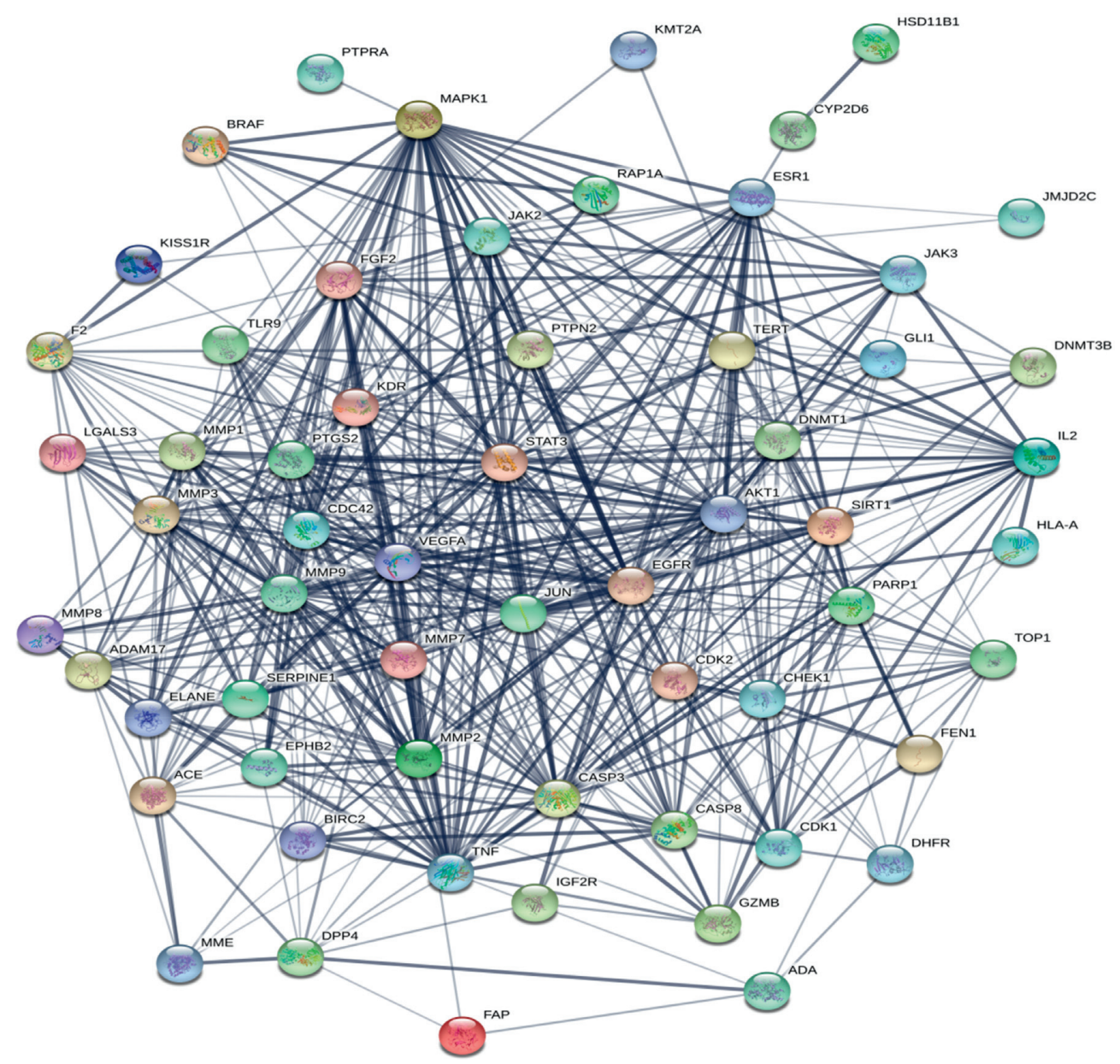

(a)

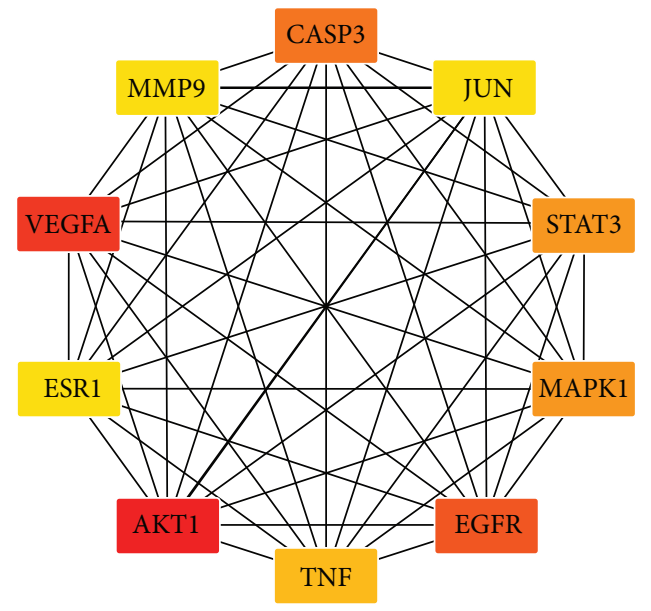

(b)

Figure 3: (a) Target protein interaction network (PPI). (b) The top 10 hub genes. The darker the color, the higher the score and the stronger the connection.

MSCs in vivo. The ESR1 and ESR2 genotypes are associated with insulin sensitivity and metabolic syndrome in Japanese and Chinese women. The ESR1 gene is important in preventing and reducing inflammation and glucose tolerance $[24,25]$. In the pathogenesis of diabetic wounds, the expression level of MMP9 increases, impairing the balance of
ECM synthesis, and degradation can cause the wound to be difficult to heal. In addition, the expression level of MMP9 gradually decreases as the wound heals [26, 27]. Therefore, the extract of Periplaneta americana may promote the healing of diabetic anal fistula wounds through multicomponent regulation and multitarget synergy. 


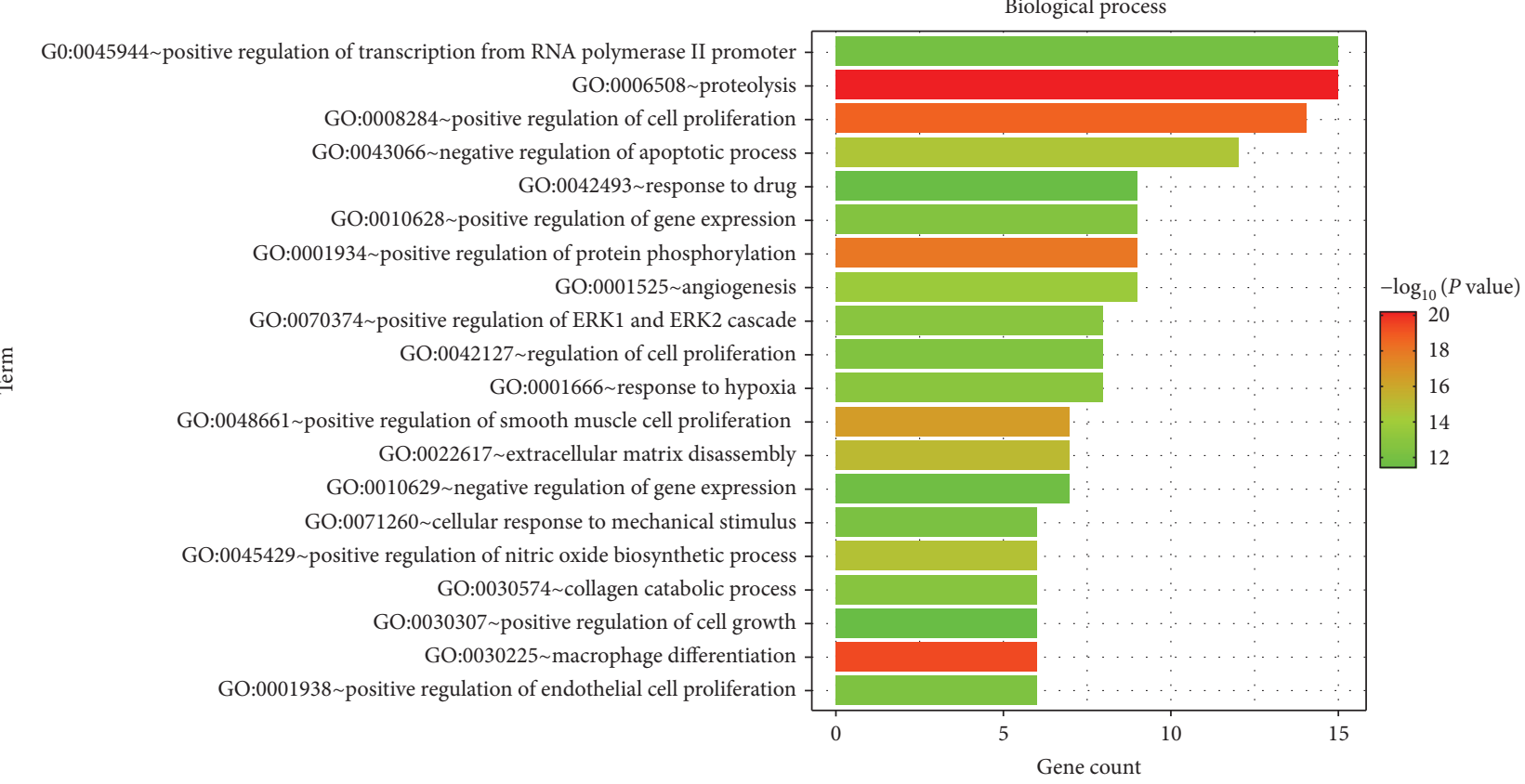

(a)

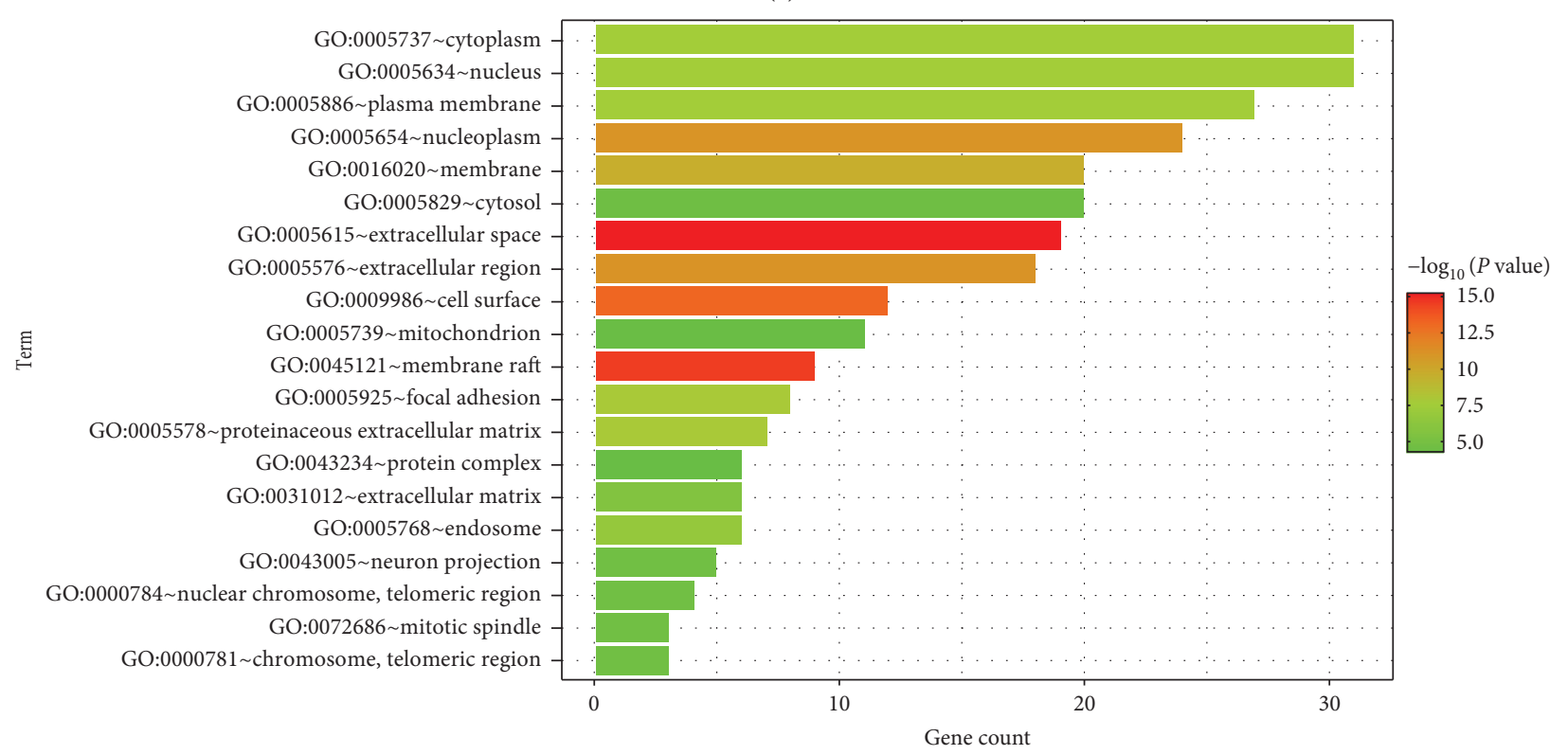

(b)

Figure 4: Continued. 


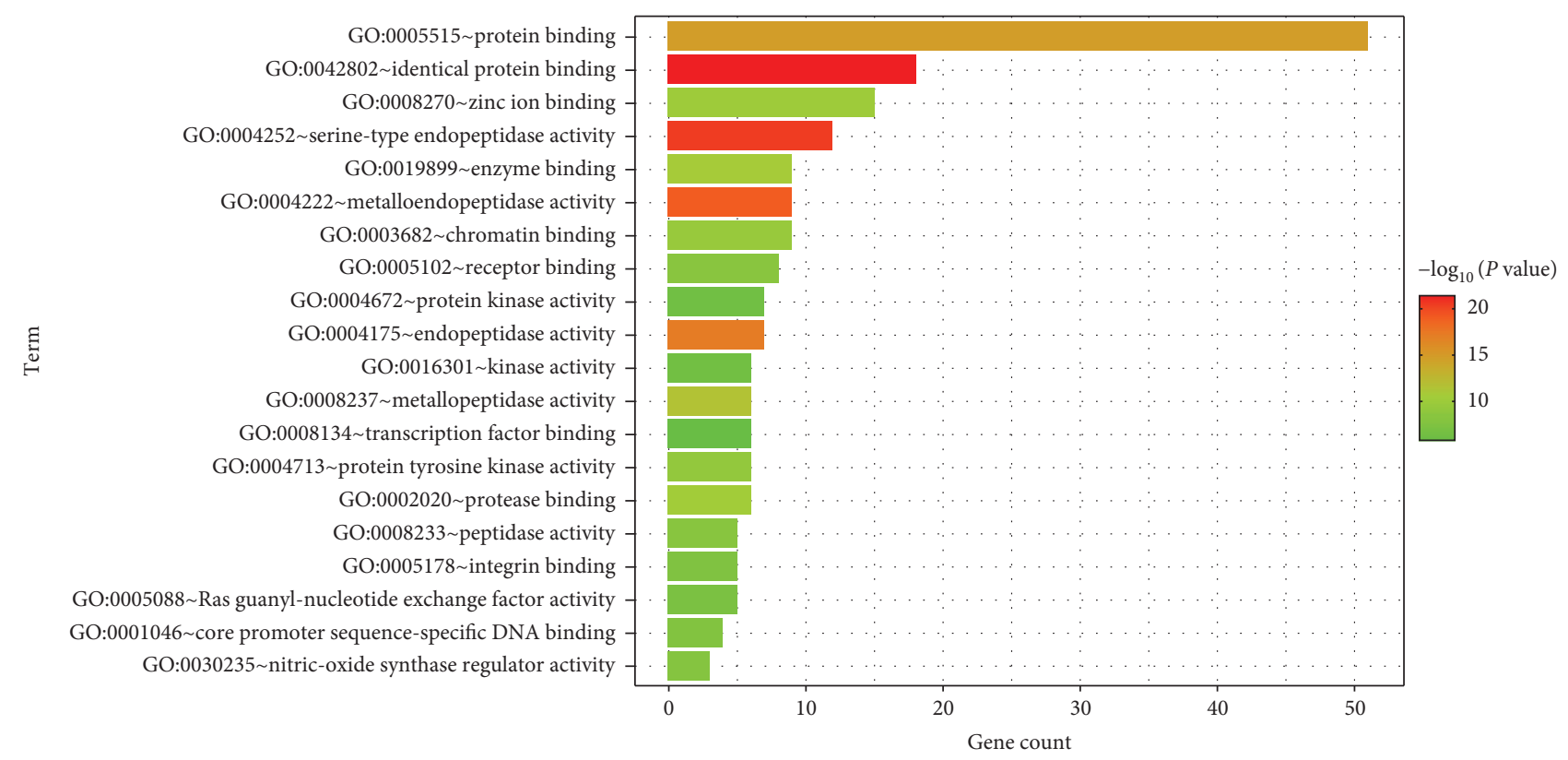

(c)

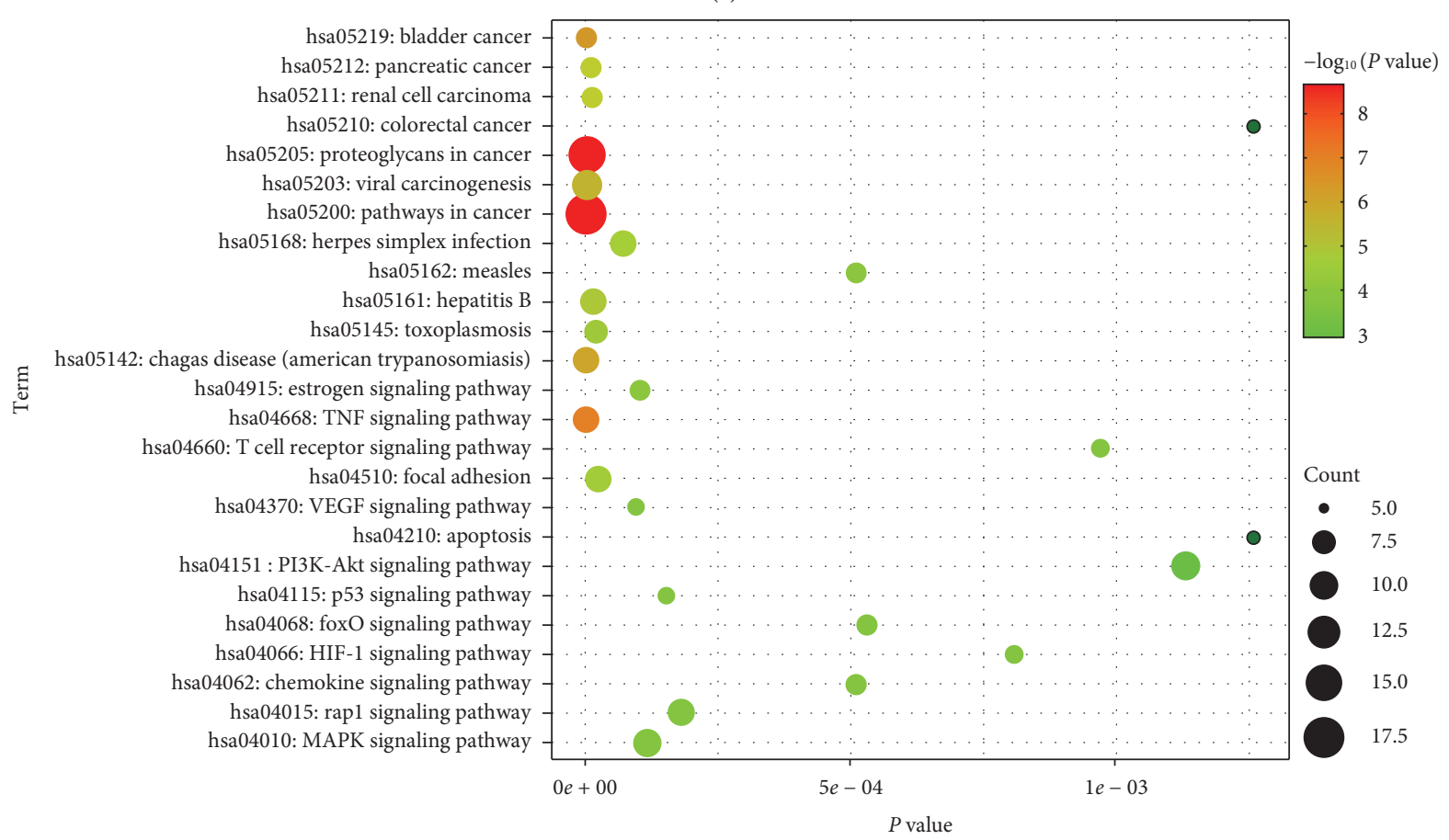

(d)

FIGURE 4: (a) Biological process, (b) cell component, (c) molecular function, and (d) KEGG pathway enrichment analysis.

GO enrichment analysis found that the extract of Periplaneta americana mainly promoted wound healing after diabetic anal fistula surgery through biological processes such as cell proliferation, macrophage differentiation, angiogenesis, and response to hypoxia. Studies have found that the macrophage subgroup Ly6CHi cells show a peak of MCP-1 in diabetic wounds. However, the Ly6CHi influx is reversed by the selective administration of anti-MCP-1, thus improving wound healing [28]. In vitro studies have shown [22] that a high glucose environment is conducive to the polarization of M1 type macrophages and produces a large amount of TNF- $\alpha$, which is not conducive to the migration of keratinocytes and affects wound healing. Through the analysis of the KEGG pathway, it was found that the main enriched PI3K-Akt signaling pathway, HIF-1 signaling pathway, and estrogen signaling pathway may play an important role in diabetic anal fistula wounds. Epidemiological studies have shown that the occurrence of the anal fistula may be closely related to sex hormone levels and anal gland infection [29]. The genes enriched in the 
estrogen signaling pathway are JUN, MMP2, MAPK1, AKT1, ESR1, MMP9, and EGFR. Except for MMP2, the rest belong to the top 10 key genes in the PPI network. El-Tawil found that the rate of women suffering from congenital fistula is lower than that of men. Estrogen has anti-inflammatory activity and can antagonize the stimulation of the lipopolysaccharide component (LPS) of the cell wall of Gram-negative bacteria. Studies have confirmed that estrogen can accelerate wound healing, by reducing wound macrophage infiltration, inhibiting LPS-induced inflammatory signals, promoting the migration of mouse keratinocytes in vitro, promoting wound formation in diabetic mice, improving insulin sensitivity, and stimulating glucose to ingest [30-33]. Estrogen may become a new therapeutic target for diabetic anal fistula wounds. Clinical studies have found that, with the aggravation of male diabetic foot ulcers, the level of estradiol in the body increased and the level of testosterone decreased [34]. J. Xia et al. [35] found that the androgen level of anal fistula patients was higher than that of patients with nonanal fistula, which may be related to the rapid proliferation of the anal glands caused by the elevated testosterone level of patients. The role of sex hormones on the wounds of diabetic anal fistula surgery remains to be confirmed by further studies.

\section{Conclusion}

In summary, Periplaneta americana extracts may participate in biological processes such as macrophage differentiation, angiogenesis, and response to hypoxia. Regulation of PI3KAkt signaling pathway, HIF-1 signaling pathway, and estrogen signaling pathway promotes wound healing. This study clarified the relationship between the main components, targets, pathways, and diseases of the American cockroach extract through network pharmacology techniques and approaches and elucidated that it can promote wound healing through multiple targets and multiple pathways. The above findings provide an important theoretical basis for further experimental research.

\section{Data Availability}

The data used to support the findings of this study are included within the article.

\section{Conflicts of Interest}

The authors declare that they have no conflicts of interest.

\section{Acknowledgments}

This research was funded by the National Natural Science Foundation of China (no. 81774325).

\section{References}

[1] S. X. Song, "Guidelines for the clinical diagnosis and treatment of perianal abscess, anal fistula, and rectovaginal fistula of the American College of Colorectal Surgeons," Chinese
Journal of Gastrointestinal Surgery, vol. 20, no. 12, pp. 14371439, 2017.

[2] P. Saeedi, I. Petersohn, P. Salpea et al., "Global and regional diabetes prevalence estimates for 2019 and projections for 2030 and 2045: results from the international diabetes federation diabetes atlas, 9th edition," Diabetes Research and Clinical Practice, vol. 157, Article ID 107843, 2019.

[3] Z.-Q. Ma, X.-Y. Huang, and J. He, "Analysis of risk factors affecting the quality of wound healing after perianal abscess or anal fistula," Clinical Misdiagnosis and Mistreatment, vol. 33, no. 2, pp. 76-80, 2020.

[4] S.-L. Lu, T. Xie, Y.-W. Niu et al., "A potential mechanism for impaired wound healing-cutaneous environmental disorders in diabetes mellitus," Chinese Journal of Burns, vol. 24, no. 1, pp. 3-5, 2008.

[5] D. Wang, G. Yang, J. Qiu et al., "Risk factors for anal fistula: a case-control study," Techniques in Coloproctology, vol. 18, no. 7, pp. 635-639, 2014.

[6] Y. Yan, X. Liu, Y. Zhuang et al., "Pien Tze Huang accelerated wound healing by inhibition of abnormal fibroblast apoptosis in Streptozotocin induced diabetic mice," Journal of Ethnopharmacology, vol. 261, p. 113203, 2020.

[7] Y. Qu, Z. Zhang, Y. Lu et al., "Network pharmacology reveals the molecular mechanism of cuyuxunxi prescription in promoting wound healing in patients with anal fistula," $E v$ idence-Based Complementary and Alternative Medicine, vol. 2019, Article ID 3865121, 9 pages, 2019.

[8] S. H. Mosavat, L. Ghahramani, E. R. Haghighi et al., "Anorectal diseases in Avicenna's "canon of medicine," Acta medico-historica adriatica, vol. 13, no. 2, pp. 103-114, 2015.

[9] H. Yang, D. Gan, Y. Li et al., "Quyushengxin formula causes differences in bacterial and phage composition in ulcerative colitis patients," Evidence-Based Complementary and Alternative Medicine, vol. 2020, Article ID 5859023, 13 pages, 2020.

[10] M. H. Hashempur, F. Khademi, M. Rahmanifard, and M. M. Zarshenas, "An evidence-based study on medicinal plants for hemorrhoids in medieval persia," Journal of Evidence-Based Complementary \& Alternative Medicine, vol. 22, no. 4, pp. 969-981, 2017.

[11] J. J. Zhu, S. Yao, X. Guo et al., "Bioactivity-guided screening of wound-healing active constituents from American cockroach (Periplaneta americana)," Molecules, vol. 23, no. 1, 2018.

[12] N. Lv, J.-C. Wang, L.-H. Chen et al., "Establishment of HPLC fingerprint of Kangfu Xinye and determination of $6 \mathrm{com}-$ ponents," Chinese Patent Medicine, vol. 40, no. 3, pp. 613-617, 2018.

[13] W.-M. Wu, L.-Y. Jin, Z.-Q. Wang et al., "Clinical study on Kangfuxin liquid in treatment of anal difficult to heal chronic wounds," Guiding Journal of Traditional Chinese Medicine and Pharmacy, vol. 21, no. 22, pp. 71-73, 2015.

[14] D.-X. Zhao, L. Yu, and L.-B. Wang, "Observation of the curative effect of Kangfuxin liquid in patients with diabetic perianal abscess after operation," Diabetes New World, vol. 19, no. 22, pp. 64-65, 2016.

[15] A. L. Hopkins, "Network pharmacology," Nature Biotechnology, vol. 25, no. 10, pp. 1110-1111, 2007.

[16] X.-W. Ma, Z.-J. Chen, X. Li et al., "Simultaneous determination of 9 nucleoside components in Periplaneta americana extract by HPLC," Traditional Chinese Medicine and Clinical Medicine, vol. 9, no. 5, pp. 1-24, 2018.

[17] J. Zeng, W. Li, L. Xiao et al., "Research on the regulation mechanism for cell apoptosis, proteoglycan and hyaluronic acid of chronic wound by the American cockroach extraction," World Science and Technology/Modernization of 
Traditional Chinese Medicine and Materia Medica, vol. 20, no. 7, pp. 1226-1231, 2018.

[18] J. Zeng, L. I. U. Xiao, W. Wu et al., "On effect of American Periplaneta extract on VEGF's gene expression of rats 'chronic refractory wound," Chinese Journal of Ethnomedicine and Ethnopharmacy.vol. 25, no. 19, pp. 64-66, 2016.

[19] M. Yang, G. E. N. G. Yue-fei, Y.-mei Shen et al., "Effects of American cockroach extracting solution on expression of Smad6, Smad9 in TGF- $\beta 1$ signal pathway in wound healing and pathology ultrastructural observation," CJTCMP, vol. 34, no. 07, pp. 3266-3269, 2019.

[20] W. Li, W. Wu, L. Xiao et al., "Study of American cockroach extraction solution in regulating the Type I/III collagen of chronic wound," Journal of Hunan University of Chinese Medicine, vol. 38, no. 3, pp. 250-253, 2018.

[21] H. B. Lindner, A. Zhang, J. Eldridge et al., "Anti-bacterial effects of poly-N-acetyl-glucosamine nanofibers in cutaneous wound healing: requirement for Akt1," PLoS One, vol. 6, no. 4, Article ID e18996, 2011.

[22] S.-M. Huang, C.-S. Wu, M.-H. Chiu et al., "High glucose environment induces M1 macrophage polarization that impairs keratinocyte migration via TNF- $\alpha$ : an important mechanism to delay the diabetic wound healing," Journal of Dermatological Science, vol. 96, no. 3, pp. 159-167, 2019.

[23] C. Yue, Z. Guo, Y. Luo et al., "c-Jun overexpression accelerates wound healing in diabetic rats by human umbilical cordderived mesenchymal stem cells," Stem Cells International, vol. 2020, Article ID 7430968, 10 pages, 2020.

[24] J. C. Lo, X. Zhao, A. Scuteri, S. Brockwell, and M. R. Sowers, "The association of genetic polymorphisms in sex hormone biosynthesis and action with insulin sensitivity and diabetes mellitus in women at midlife," The American Journal of Medicine, vol. 119, no. 9, pp. S69-S78, 2006.

[25] K. Moore, G. Ghatnekar, R. G. Gourdie et al., "Impact of the controlled release of a connexin 43 peptide on corneal wound closure in an STZ model of type I diabetes," PLoS One, vol. 9, no. 1, Article ID e86570, 2014.

[26] W. Wang, C. Yang, X. y. Wang et al., "MicroRNA-129 and -335 promote diabetic wound healing by inhibiting sp1mediated MMP-9 expression," Diabetes, vol. 67, no. 8, pp. 1627-1638, 2018.

[27] T. T. Nguyen, D. Ding, W. R. Wolter et al., "Validation of matrix metalloproteinase-9 (MMP-9) as a novel target for treatment of diabetic foot ulcers in humans and Discovery of a potent and selective small-molecule MMP-9 inhibitor that accelerates healing," Journal of Medicinal Chemistry, vol. 61, no. 19, pp. 8825-8837, 2018.

[28] A. Kimball, M. Schaller, A. Joshi et al., "Ly6CHiBlood monocyte/macrophage drive chronic inflammation and impair wound healing in diabetes mellitus," Arteriosclerosis, Thrombosis, and Vascular Biology, vol. 38, no. 5, pp. 11021114, 2018.

[29] A.-Q. Zhao, A Preliminary Study on the Risk Factors of Anal Glandular Infectious Diseases, Chengdu University of Traditional Chinese Medicine, Chinese Surgery (Anorectal), Chengdu, China, 2019.

[30] A. M. El-Tawil, "Mechanism of non-specific-fistula-in-ano: hormonal aspects-Review," Pathophysiology, vol. 19, no. 1, pp. 55-59, 2012.

[31] R. Crompton, H. Williams, D. Ansell et al., "Oestrogen promotes healing in a bacterial LPS model of delayed cutaneous wound repair," Laboratory Investigation, vol. 96, no. 4, pp. 439-449, 2016.
[32] Y. Zhuge, M. M. Regueiro, R. Tian et al., "The effect of estrogen on diabetic wound healing is mediated through increasing the function of various bone marrow-derived progenitor cells," Journal of Vascular Surgery, vol. 68, no. 6, pp. 127S-135S, 2018.

[33] R. S. Campello, L. A. Fátima, J. N. Barreto-Andrade et al., "Estradiol-induced regulation of GLUT4 in 3T3-L1 cells: involvement of ESR1 and AKT activation," Journal of Molecular Endocrinology, vol. 59, no. 3, pp. 257-268, 2017.

[34] Y. He, L. Xu, H.-J. Qian et al., "The changes of pituitary-gonad axis function in men with diabetic foot ulcers and its correlation with prognosis," Chinese Journal of Endocrinology and Metabolism, vol. 1, pp. 23-27, 2015.

[35] J.-Y. Xia, D.-W. Liu, B. Cao et al., "Research on the correlation factors of male testosterone and the causes of anal fistula," Colorectal Surgery, vol. 4, pp. 437-439, 2016. 